\title{
REFERENCES
}

1. N. Aronszajn, Sur quelques problèmes concernant les espaces de Minkowski et les espaces vectoriels généraux, Atti della Accademia Nazionale dei Lincei. Rendiconti (6) vol. 26 (1937) pp. 374-376.

2. Stefan Banach, Theorie des operations lineaires, Warsaw, 1932.

3. F. F. Bonsall, $A$ note on subadditive functionals, J. London Math. Soc. vol. 29 (1954) pp. 125-126.

4. N. Bourbaki, Espaces vectoriels topologiques, Actualités Scientifiques et Industrielles, no. 1189, Paris, 1953.

5. V. L. Klee, Jr., Convex sets in linear spaces, Duke Math. J. vol. 18 (1951) pp. 443-466, 875-883.

6. Y. Tagamlitzki, Übertragung des Minkowskischen Stiutzebenensatzes auf Hilbertsche Räume, C. R. Acad. Bulgare Sci. vol. 4 (1951) no. 2-3 (1953) pp. 5-8.

UNIVERSITY OF WASHINGTON

\section{A NOTE ON HAAR MEASURE}

\section{HARRY F. DAVIS}

We prove a direct sum theorem for Haar measure which is a generalization, to arbitrary locally compact topological groups, of a theorem for Lie groups due to Mostow [3]. It is well known that the Haar integral on a compact group may be obtained by a simple averaging process. On a vector group, the Haar integral is essentially the Lebesgue integral. On a discrete group it is summation. Our theorem asserts that the formation of a Haar integral on any locally compact group must be a composite of these three processes. Hence no locally compact group exists for which the Haar integral must be constructed in an essentially more novel manner.

THEOREM. Given any locally compact topological group $G$, there exists a compact subgroup $K$, a subspace $E$ which is homeomorphic to an ndimensional Euclidean space, and a discrete subset $D$, such that the mapping $\theta(a, b, c)=a b c$ is a homeomorphism of the Cartesian product $D \times E \times K$ with $G$, and carries the product measure $m_{D} \times m_{E} \times m_{K}$ over to $m_{G}$. Here $m_{D}$ is discrete measure in $D, m_{E}$ is Lebesgue measure in $E$ relative to suitable coordinates, $m_{K}$ is Haar measure in $K$, and $m_{G}$ is left Haar measure in $G$. (Note that $E$ need not be a subgroup.)

Received by the editors June 18, 1954.

1 The material in this paper constitutes part of a thesis submitted to the Massachusetts Institute of Technology and prepared under the direction of Professor Kenkichi Iwasawa. 
We first prove this theorem for a group $G$ having a compact normal subgroup $N$ such that $G / N$ is a connected Lie group, making use of the fact that Mostow has proved it for Lie groups. Then we use the fact, proved by Yamabe, that any locally compact group has an open subgroup with this property. We must first prove the following lemma, which was proved for connected groups by Iwasawa [2, p. 545].

LEMMA 1. Let $G$ be a locally compact group and $N$ a compact normal subgroup of $G$. If $H_{1}$ is a closed subgroup of $G$ containing $N$ and if $H_{1} / N$ is isomorphic with the reals, then there is contained in the connected component of the identity of $G$ a closed subgroup $H$ also isomorphic with the reals such that $H_{1}=H N, H \cap N=\{e\}$.

Proof. It suffices to prove the assertion for the case $G=H_{1}$. Let $G^{0}$ be the connected component of the identity of $G$. The natural homomorphism $\pi$ of $G$ on to $G / N$ maps $G^{0}$ onto $G^{0} N / N$. Since $N$ is compact, $G^{0} N / N$ is topologically isomorphic with $G^{0} /\left(G^{0} \cap N\right)$ (see $\left[4\right.$, p. 17]), so we have a natural homomorphism $\pi^{\prime}$ of $G^{0}$ onto $G^{0} /\left(G^{0} \cap N\right)$.

Since $G^{0}$ is connected and $\pi$ is continuous, the image of $G^{0}$ under $\pi$ is a connected subgroup of $G / N$. Since $G / N$ is isomorphic with the reals, this image is either $\{e\}$ or a subgroup isomorphic with the reals. The first of these possibilities gives $G^{0} /\left(G^{0} \cap N\right) \approx\{e\}$, implying $G^{0} \subseteq N$. Then $G / N \approx\left(G / G^{0}\right) /\left(N / G^{0}\right)$ (see $[4$, p. 12]) and this is totally disconnected because $G / G^{0}$ is. This contradicts the isomorphism of $G / N$ with the reals. So we can have only the second possibility, that $G^{0} /\left(G^{0} \cap N\right)$ is isomorphic with the reals. In this case, Lemma 4.7 of [2] applies directly to give the result since $G^{0}$ is connected.

LeMmA 2. Let $G$ be a locally compact group which has a compact normal subgroup $N$ such that $G / N$ is a connected Lie group. Then the assertion of the theorem holds with $D=\{e\}$.

Proof. Theorem 1 of [3] implies that, given any connected Lie group $G^{\prime}$, there exists a compact subgroup $K^{\prime}$ and a Euclidean subspace $E^{\prime}$ such that the mapping $\theta$ of $E^{\prime} \times K^{\prime}$ onto $G^{\prime}$ defined by $\theta(a, b)=a b$ is a homeomorphism; also, $E^{\prime}=U_{1}^{\prime} U_{2}^{\prime} \cdots U_{n}^{\prime}$ where the $U_{i}^{\prime}$ are isomorphic with the reals and this decomposition is topological in the sense that the mapping $\phi\left(u_{1}, u_{2}, \cdots, u_{n}\right)=u_{1} u_{2} \cdots u_{n}$ of $U_{1}^{\prime} \times U_{2}^{\prime} \times \cdots \times U_{n}^{\prime}$ on to $E^{\prime}$ is a homeomorphism. Moreover, given any continuous function $f$ which vanishes outside a compact set,

$$
\int_{G^{\prime}} f(x) d m_{G^{\prime}}(x)=\int_{E^{\prime}} d m_{E^{\prime}} \int_{K^{\prime}} f(a b) d m_{K^{\prime}}(b)
$$


where $m_{B^{\prime}}$ is Lebesgue measure relative to the coordinates $\phi^{-1}$ and $m_{K^{\prime}}$ is Haar measure in $K^{\prime}$.

Letting $\pi$ denote the natural homomorphism of $G$ onto $G / N$, and applying Lemma 1 , we find subgroups $U_{1}, U_{2}, \cdots, U_{n}$, each isomorphic with the reals, such that $\pi^{-1}\left(U_{i}^{\prime}\right)=U_{i} N, U_{i} \cap N=\{e\}$. Denoting $\pi^{-1}\left(K^{\prime}\right)$ by $K$, we have $G=U_{1} U_{2} \cdots U_{n} K$ where $K / N=K^{\prime}$, $U_{i} \cap K=\{e\}, i=1,2, \cdots, n$. Using arguments essentially like those in [2], it is seen easily that this decomposition is topological; in particular, $E=U_{1} U_{2} \cdots U_{n}$ is homeomorphic via $\pi$ with $E^{\prime}$.

Let $f$ be any continuous function on $G$ which vanishes outside a compact set. By a theorem of Weil $[4$, p. 45],

$$
\int_{G} f d m_{G}=\int_{G / N} d m_{G / N} \int_{N} f(x n) d m_{N}(n)
$$

where $m_{\text {G/N }}$ and $m_{N}$ are left Haar measures in $G / N$ and $N$ respectively. Observing that $\int_{N} f(x n) d m_{N}(n)$ is constant on cosets of $N$ and hence defines a function $g(x)$ on $G^{\prime}=G / N$ which vanishes outside a compact set, we apply Mostow's theorem to give

$$
\int_{G} f d m_{G}=\int_{E^{\prime}} d m_{E^{\prime}}\left(a^{\prime}\right) \int_{K^{\prime}} g\left(a^{\prime} b\right) d m_{K^{\prime}}(b) .
$$

Applying Weil's theorem again, this time to $K$, we have, for any $a$ in $E$,

$$
\int_{K} f(a k) d m_{K}(k)=\int_{K^{\prime}} d m_{K^{\prime}} \int_{N} f(a c n) d m_{N}(n)=\int_{K^{\prime}} g\left(a^{\prime} b\right) d m_{K^{\prime}}(b)
$$

where $a^{\prime}=\pi a$. So if we carry $m_{E^{\prime}}$ back to $E$ via the inverse of the restriction of $\pi$ to $E$, we have a Lebesgue measure $m_{E}$ in $E$ and can write

$$
\int_{G} f d m_{G}=\int_{E} d m_{E}(a) \int_{K} f(a k) d m_{K}(k) .
$$

We have proved this formula only for functions which are continuous and have compact support, but Ambrose shows in [1] how to extend such formulas to a wider class of functions and obtain the asserted measure-theoretic fact when the Haar measures are taken to be nontrivial left-invariant Baire measures. His direct sum theorem is stated only for invariant subgroups, but nevertheless his proofs carry over to our case with little modification.

Proof of Theorem. Yamabe showed in [5] that any locally compact topological group contains an open subgroup $G^{0}$ and a compact 
normal subgroup $N \subseteq G^{0}$ such that $G^{0} / N$ is a connected Lie group. Choose representing elements from the left cosets of $G^{0}$ in $G$ to make up a set $D$, so that $G=D G^{0}$. Then $D$ is discrete in the induced topology and for integrable $f$,

$$
\int f d m_{G}=\sum_{d \in D} \int_{G^{0}} f(d x) d m_{G^{0}}(x)
$$

so the homeomorphism $\theta(d, x)=d x$ of $D \times G^{0}$ onto $G$ carries $m_{D} \times m_{G^{0}}$ onto $m_{a}$. This, together with Lemma 2, proves the theorem.

We remark in concluding that we can prove Mostow's theorem, in the weakened form in which we used it here, more directly by applying methods due to Weil [4]. We intend to discuss this, together with some applications of the theorem proved here to the theory of group representations, in a later paper.

\section{BiBLIOGRAPHY}

1. W. Ambrose, Direct sum theorem for Haar measures, Trans. Amer. Math. Soc. vol. 71 (1947) pp. 122-127.

2. K. Iwasawa, On some types of topological groups, Ann. of Math. vol. 50 (1949) pp. 507-558.

3. G. D. Mostow, On the $L^{2}$-space of a Lie group, Amer. J. Math. vol. 74 (1952) pp. 920-928.

4. A. Weil, L'integration dans les groupes topologiques et ses applications, Actualités Scientifiques et Industrielles, no. 869, Hermann, 1940.

5. H. Yamabe, $A$ generalization of a theorem of Gleason, Ann. of Math. vol. 58 (1953) pp. 351-365.

Massachusetts Institute of Technology 